$\xi=-1$

\title{
Synthesis and Characterization of Alginate Encapsulated Zirconium-Based Ferromagnetic Sorbent for Adsorptive Removal of Dyes
}

\author{
Soh Fong Lim ${ }^{1}$, Agnes Yung Weng Lee', Yat Seng Kam ${ }^{1}$, S.N. David Chua ${ }^{1}$ \\ ${ }^{1}$ Faculty of Engineering, Universiti Malaysia Sarawak, 94300 Kota Samarahan, Sarawak, Malaysia \\ *Corresponding author E-mail: sflim@unimas.my
}

\begin{abstract}
This paper provides insight on the removal of dyes from water using magnetic separation technique. A new alginate encapsulated zirconium-based encapsulated ferromagnetic sorbent has been synthesized by co-precipitation and electrostatic extrusion techniques. The ferromagnetic sorbent has the alginate as outer polymeric shell and zirconium-based iron oxides as inner ferromagnetic cores. The sorbent has the multi-functional properties as it is can be easily separated using external magnetic force, and effective in adsorptive removal of congo red, methyl violet, and methylene blue dyes. The sorbent was characterized by particle size analyser, Scanning Electron Microscope (SEM), Thermal Gravimetric-Differential Thermal Analyzer (TG-DTA), and Fourier Transform Infrared Spectroscopy (FTIR). Adsorption assays were performed in batch using methyl violet, methylene blue, and congo red dyes as contaminants. The alginate encapsulated zirconium-based ferromagnetic sorbent was a potential candidate for removal of dyes under a magnetic field as separating agent. The present investigation demonstrates the ferromagnetic sorbent exhibiting good performance to remove the methyl violet, congo red, and methylene blue dyes from aqueous solutions. The adsorption experiments reveal that the adsorption performance is higher for the cationic dye than the anionic dye. The SEM and FT-IR studies show that the interaction characteristics between the dyes and the ferromagnetic sorbent where the surface of the ferromagnetic sorbent became smoother and less porous when the metal-O group ( $\mathrm{Zr}-\mathrm{O}$ and $\mathrm{Fe}-\mathrm{O})$ of the ferromagnetic sorbent attaches to the dye.
\end{abstract}

Keywords: Adsorption; Congo red; Dyes; Magnetic separation; Methyl blue; Methyl violet.

\section{Introduction}

Dyes which are widely used in painting, textiles, printing, cosmetic, laundry, synthesis, tannery, pulp mill, leather, and food industries are one of the common organic contaminants in wastewater. Congo red dye has anionic property whereas methylene blue and methyl violet dyes are both cationic charges in nature [1]. These dyes are considered as hazardous contaminants due to their toxicity to living organisms. Therefore, these dyes in effluents of wastewater streams have to be sufficiently treated before they are discharged into water bodies. Based on previous findings from other researchers, adsorption process is one of the most efficient methods used for decontaminant of the dyes [2].

Magnetic adsorbents which have high catalytic activity and are easily separated from water system have advantageous over the traditional sorbent materials in practical application as sorbent. More specifically, the excellent ferromagnetic property has enabled easy recovery of the materials by magnetic separation technique after decontamination of contaminants, which overcomes the difficulty of separation in common adsorbents [3].

In the same way, the magnetic iron oxide particles exhibit amphoteric surface activity, easy dispersion ability, and their very small size and dimensions, a high surface-to-volume ratio, resulting in a high adsorption capacity [4]. Furthermore, the magnetite, $\mathrm{Fe}_{3} \mathrm{O}_{4}$ is known to have low toxicity and good biocompatibility, which is beneficial for water treatment in practice. Other elements such as
$\mathrm{Mn}, \mathrm{Ce}, \mathrm{Zr}$, and $\mathrm{Al}$ have been incorporated and integrated with iron oxides to prepare bimetal oxide composites to enhance the performance of adsorption process [5]. In this study, zirconium based ferromagnetic particles were encapsulated by calcium alginate through electrostatic extrusion to produce an innovative ferromagnetic sorbent. As this ferromagnetic sorbent has both zirconium-based ferromagnetic particles (which consist of $\mathrm{Zr}, \mathrm{Fe}$, and $\mathrm{O}$ elements) and calcium alginate, it demonstrates multi-functional adsorption properties for both anionic and cationic contaminants for dyes removal.

\section{Materials and Method}

\subsection{Materials}

All chemicals used in this study were of analytical reagent grade. The materials that were used for sorbent synthesization were iron (II) chloride tetrahydrated $\left(\mathrm{FeCl}_{2} \cdot 4 \mathrm{H}_{2} \mathrm{O}\right)$ of $99 \%$ purity (SigmaAldrich, France), iron (III) chloride $\left(\mathrm{FeCl}_{3}\right)$ (greater than $97 \%$, Sigma-Aldrich, France), distilled water, sodium hydroxide solution $(\mathrm{NaOH})(50 \%$ solution in water, Sigma-Aldrich, France), sodium alginate $\left(\mathrm{C}_{5} \mathrm{H}_{7} \mathrm{O}_{4} \mathrm{COONa}\right)(99.999 \%$, Aldrich, U.S.A), zirconium (IV) sulfate $\left(\mathrm{Zr}\left(\mathrm{SO}_{4}\right)_{2}\right](35$ weight \% solution in water, $99.9 \%$, Aldrich, U.S.A) and calcium chloride dihydrated $\left(\mathrm{CaCl}_{2} \cdot 2 \mathrm{H}_{2} \mathrm{O}\right)(99.9 \%$, Merck, Germany). The stock solution dyes were prepared by dissolving methyl violet (99.999\%, Fluka, India), methylene blue hydrate $(99.999 \%$, Fluka, India), and congo red 